Universidad Católica del Norte

Antofagasta - Chile

\title{
Spectral analysis of Hahn-Dirac system
}

\author{
B. P. Allahverdiev \\ Süleyman Demirel University, Turkey \\ and \\ Hüseyin Tuna \\ Mehmet Akif Ersoy University, Turkey \\ Received: April 2021. Accepted : June 2021
}

\begin{abstract}
In this paper, we study some spectral properties of the one-dimensional Hahn-Dirac boundary-value problem, such as formally self-adjointness, the case that the eigenvalues are real, orthogonality of eigenfunctions, Green's function, the existence of a countable sequence of eigenvalues, eigenfunctions forming an orthonormal basis of $L_{\omega, q}^{2}\left(\left(\omega_{0}, a\right) ; E\right)$.
\end{abstract}

2010 Mathematical Subject Classificition: $39 A 13$, 39A70, 33D15, 34B27, 34L10, 34L40, $47 A 10$.

Key words and phrases. Hahn-Dirac system, self-adjoint operator, eigenvalues and eigenfunctions, Green's matrix, eigenfunction expansion. 


\section{Introduction}

$(1.1)\left(\begin{array}{cc}0 & -1 \\ 1 & 0\end{array}\right)\left(\begin{array}{l}y_{1}^{\prime}(x) \\ y_{2}^{\prime}(x)\end{array}\right)+\left(\begin{array}{cc}p(x) & 0 \\ 0 & r(x)\end{array}\right)\left(\begin{array}{l}y_{1}(x) \\ y_{2}(x)\end{array}\right), x \in[a, b]$,

where $p($.$) and r($.$) are real-valued, Lebesgue integrable functions on [a, b]$, is one of the classical equations in quantum mechanics. This system predicts the existence of antimatter and describes the electron spin. Some properties of the one-dimensional Dirac systems have been considered in the literature (see $[19,24,23,1]$ and the references therein).

The Hahn difference operator $D_{\omega, q}$ defined by $(q \in(0,1), \omega>0)$

$$
\mathrm{D}_{\omega, q} f(x)=\left\{\begin{array}{cc}
\frac{f(\omega+q x)-f(x)}{\omega+(q-1) x}, & x \neq \omega_{0}, \\
f^{\prime}\left(\omega_{0}\right), & x=\omega_{0},
\end{array}\right.
$$

was introduced by Hahn [9], [10]. This operator is known as a generalization of the forward difference operator defined by

$$
\Delta_{\omega} f(x):=\frac{f(\omega+x)-f(x)}{(\omega+x)-x}, x \in \mathbf{R} .
$$

and the quantum $q$-difference operator ([15]) defined by

$$
D_{q} f(x):=\frac{f(q x)-f(x)}{q x-x}, x \neq 0 .
$$

Hahn difference operators are receiving an increase of interest due to their applications in the construction of families of orthogonal polynomials and approximation problems see [5],[8], [17], [18], [21] and the references therein.

In the literature there exist some papers including Hahn difference equations. In [12], Hamza et al. studied the theory of linear Hahn difference equations. The authors also study the existence and uniqueness of solutions for the initial value problems for Hahn difference equations. Moreover, they proved Gronwall's and Bernoulli's inequalities with respect to the Hahn difference operator and investigated the mean value theorems for this calculus. In 2016, Hamza and Makharesh [13] investigated Leibniz's rule and Fubini's theorem associated with the Hahn difference operator. Sitthiwirattham [22] considers the nonlocal boundary value problem for the nonlinear Hahn difference equation. Recently, in [7], the regular HahnSturm-Liouville problem

$$
-q^{-1} D_{-\omega q^{-1}, q^{-1}} D_{\omega, q} y(x)+v(x) y(x)=\lambda y(x),
$$




$$
\begin{aligned}
a_{1} y\left(\omega_{0}\right)+a_{2} D_{-\omega q^{-1}, q^{-1}} y\left(\omega_{0}\right) & =0, \\
b_{1} y(b)+b_{2} D_{-\omega q^{-1}, q^{-1}} y(b) & =0,
\end{aligned}
$$

is studied where $\omega_{0} \leq x<\infty, \lambda \in \mathbf{C}, a_{i}, b_{i} \in \mathbf{R}:=(-\infty, \infty), i=1,2$, and $p($.$) is a real-valued continuous function at \omega_{0}$ defined on $\left[\omega_{0}, b\right]$. Annaby et al. [7] define a Hilbert space of $\omega, q$-square summable functions. They discussed the formulation of the self-adjoint operator and the properties of the eigenvalues and the eigenfunctions. Furthermore, they constructed Green's function and gave an eigenfunction expansion theorem.

In [14], the author introduces $\omega, q$-analogy of the Dirac system (1.1)

$$
\begin{gathered}
\Gamma y:=\left\{\begin{array}{c}
-q^{-1} D_{-\omega q^{-1}, q^{-1}} y_{2}+p(x) y_{1}, \\
D_{\omega, q} y_{1}+r(x) y_{2}
\end{array}\right. \\
\Gamma y=\lambda y, y=\left(\begin{array}{c}
y_{1} \\
y_{2}
\end{array}\right), \omega_{0}<x<a<\infty,
\end{gathered}
$$

with boundary conditions

$$
\begin{aligned}
k_{11} y_{1}\left(\omega_{0}\right)+k_{12} y_{2}\left(\omega_{0}\right) & =0, \\
k_{21} y_{1}(a)+k_{22} y_{2}\left(h^{-1}(a)\right) & =0,
\end{aligned}
$$

where $\lambda$ is a complex eigenvalue parameter, $k_{i j} \in \mathbf{R}(i, j=1,2)$ and $p($.$) ,$ $r($.$) are real-valued continuous functions at \omega_{0}$, defined on $\left[\omega_{0}, a\right]$. Hira investigated the existence and uniqueness of solutions for this problem and gave its spectral properties. Recently, in $[2,3]$, the authors proved the existence of a spectral function for the singular Hahn-Dirac system.

In this paper, we study some spectral properties of the one-dimensional Hahn-Dirac boundary-value problem (1.2), such as formally self-adjointness, the case that the eigenvalues are real, orthogonality of eigenfunctions, Green's function, the existence of a countable sequence of eigenvalues, eigenfunctions forming an orthonormal basis of $L_{\omega, q}^{2}\left(\left(\omega_{0}, a\right) ; E\right)$.

The paper is organized as follows. In preparation for our work on $\omega, q$-calculus, we first present some basic definitions and notational information in Section 2. In Section 3, we formulate a self-adjoint Hahn-Dirac system in $L_{\omega, q}^{2}\left(\left(\omega_{0}, a\right) ; E\right)$. In the last section, we construct the associated Green function of the Hahn-Dirac equation and give the eigenfunction expansions.

\section{Preliminaries}

In this section, we recall some necessary concepts of the Hahn calculus. For more details, the reader may want to consult [6], [9], [10], [7]. Throughout 
the paper, we let $q \in(0,1)$ and $\omega>0$.

Define $\omega_{0}:=\omega /(1-q)$ and let $I$ be a real interval containing $\omega_{0}$.

Definition 1 ([9],[10]). Let $f: I \rightarrow \mathbf{R}$ be a function. The Hahn difference operator is defined by

$$
D_{\omega, q} f(x)=\left\{\begin{array}{cl}
\frac{f(\omega+q x)-f(x)}{\omega+(q-1) x}, & x \neq \omega_{0}, \\
f^{\prime}\left(\omega_{0}\right), & x=\omega_{0},
\end{array}\right.
$$

provided that $f$ is differentiable at $\omega_{0}$. In this case, we call $D_{\omega, q} f$, the $\omega, q$-derivative of $f$.

Remark 2. The Hahn difference operator unifies two well-known operators. When $q \rightarrow 1$, we get the forward difference operator, which is defined by

$$
\Delta_{\omega} f(x):=\frac{f(\omega+x)-f(x)}{(\omega+x)-x}, x \in \mathbf{R} .
$$

When $\omega \rightarrow 0$, we get the Jackson $q$ - difference operator, which is defined by

$$
D_{q} f(x):=\frac{f(q x)-f(x)}{(q x)-x}, x \neq 0 .
$$

Furthermore, under appropriate conditions, we have

$$
\omega \rightarrow 0 \lim _{q \rightarrow 1} D_{\omega, q} f(x)=f^{\prime}(x) .
$$

In what follows, we present some important properties of the $\omega, q$-derivative.

Theorem 3 ([6]). Let $f, g: I \rightarrow \mathbf{R}$ be $\omega, q$-differentiable at $x \in I$ and $h(x):=\omega+q x$, then we have for all $x \in I$ :

i) $D_{\omega, q}(a f+b g)(x)=a D_{\omega, q} f(x)+b D_{\omega, q} g(x), a, b \in I$,

ii) $D_{\omega, q}(f g)(x)=D_{\omega, q}(f(x)) g(x)+f(\omega+x q) D_{\omega, q} g(x)$,

iii) $D_{\omega, q}\left(\frac{f}{g}\right)(x)=\frac{D_{\omega, q}(f(x)) g(x)-f(x) D_{\omega, q} g(x)}{g(x) g(\omega+x q)}$,

iv) $D_{\omega, q} f\left(h^{-1}(x)\right)=D_{-\omega q^{-1}, q^{-1}} f(x), h^{-1}(x)=q^{-1}(x-\omega)$

The concept of the $\omega, q$-integral of the function $f$ can be defined as follows. 
Definition 4 (Jackson-Nörlund Integral [6]). Let $f: I \rightarrow \mathbf{R}$ be a function and $a, b, \omega_{0} \in I$. We define $\omega, q$-integral of the function $f$ from $a$ to $b$ by

$$
\int_{a}^{b} f(x) d_{\omega, q}(x):=\int_{\omega_{0}}^{b} f(x) d_{\omega, q}(x)-\int_{\omega_{0}}^{a} f(x) d_{\omega, q}(x),
$$

where

$$
\int_{\omega_{0}}^{x} f(x) d_{\omega, q}(x):=((1-q) x-\omega) \sum_{n=0}^{\infty} q^{n} f\left(\omega \frac{1-q^{n}}{1-q}+x q^{n}\right), x \in I
$$

provided that the series converges at $x=a$ and $x=b$. In this case, $f$ is called $\omega, q$-integrable on $[a, b]$.

The following properties of $\omega, q$-integration can be found in [6].

Theorem 5 ([6]). Let $f, g: I \rightarrow \mathbf{R}$ be $\omega, q$-integrable on $I, a, b, c \in I$, $a<c<b$ and $\alpha, \beta \in \mathbf{R}$. Then the following formulas hold:
i) $\int_{a}^{b}\{\alpha f(x)+\beta g(x)\} d_{\omega, q}(x)=\alpha \int_{a}^{b} f(x) d_{\omega, q}(x)+\beta \int_{a}^{b} g(x) d_{\omega, q}(x)$,
ii) $\int_{a}^{a} f(x) d_{\omega, q}(x)=0$,
iii) $\int_{a}^{b} f(x) d_{\omega, q}(x)=\int_{a}^{c} f(x) d_{\omega, q}(x)+\int_{c}^{b} f(x) d_{\omega, q}(x)$,
iv) $\int_{a}^{b} f(x) d_{\omega, q}(x)=-\int_{b}^{a} f(x) d_{\omega, q}(x)$.

Next, we present the $\omega, q$-integration by parts.

Lemma 6 ([6]). Let $f, g: I \rightarrow \mathbf{R}$ be $\omega, q$-integrable on $I, a, b \in I$, and $a<b$. Then the following formula holds:

$$
\begin{aligned}
& \int_{a}^{b} f(x) D_{\omega, q} g(x) d_{\omega, q}(x)+\int_{a}^{b} g(\omega+q x) D_{\omega, q} f(x) d_{\omega, q}(x) \\
= & f(b) g(b)-f(a) g(a) .
\end{aligned}
$$

The next result is the fundamental theorem of the Hahn calculus. 
Theorem 7 ([6]). Let $f: I \rightarrow \mathbf{R}$ be continuous at $\omega_{0}$. Define

$$
F(x):=\int_{\omega_{0}}^{x} f(t) d_{\omega, q}(t), x \in I .
$$

Then $F$ is continuous at $\omega_{0}$. Moreover, $D_{\omega, q} F(x)$ exists for every $x \in I$ and $D_{\omega, q} F(x)=f(x)$. Conversely,

$$
\int_{a}^{b} D_{\omega, q} F(x) d_{\omega, q}(x)=f(b)-f(a) .
$$

Let $L_{\omega, q}^{2}\left(\omega_{0}, a\right)$ be the space of all complex-valued functions defined on $\left[\omega_{0}, a\right]$ such that

$$
\|f\|:=\left(\int_{\omega_{0}}^{a}|f(x)|^{2} d_{\omega, q} x\right)^{1 / 2}<\infty .
$$

The space $L_{\omega, q}^{2}\left(\omega_{0}, a\right)$ is a separable Hilbert space with the inner product

$$
(f, g):=\int_{\omega_{0}}^{a} f(x) \overline{g(x)} d_{\omega, q} x, f, g \in L_{\omega, q}^{2}\left(\omega_{0}, a\right)
$$

$($ see $[6])$.

\section{Self-adjoint Hahn-Dirac system}

In this section, we formulate a self-adjoint Hahn-Dirac system in $L_{\omega, q}^{2}\left(\left(\omega_{0}, a\right) ; E\right)$ and give some spectral properties of this system.

Now, we consider the Hahn-Dirac system

$$
\Gamma(y):=\left\{\begin{array}{c}
-q^{-1} D_{-\omega q^{-1}, q^{-1}} y_{2}+p(x) y_{1}, \\
D_{\omega, q} y_{1}+r(x) y_{2}
\end{array}\right.
$$

$\Gamma(y)=\lambda y, y=\left(\begin{array}{l}y_{1} \\ y_{2}\end{array}\right), \omega_{0}<x<a<\infty$,

with boundary conditions

$$
\begin{aligned}
& \Gamma_{1}(y):=a_{11} y_{1}\left(\omega_{0}\right)+a_{12} y_{2}\left(\omega_{0}\right)=0, \\
& \Gamma_{2}(y):=a_{21} y_{1}(a)+a_{22} y_{2}\left(h^{-1}(a)\right)=0,
\end{aligned}
$$

where $\lambda$ is a complex eigenvalue parameter, $a_{i j}(i, j=1,2)$ are arbitrary real numbers, $p($.$) and r($.$) are real-valued continuous functions at \omega_{0}$, defined on $\left[\omega_{0}, h^{-1}(a)\right], h^{-1}(a)=q^{-1}(a-\omega)>a$. 
We introduce convenient Hilbert space $\left.L_{\omega, q}^{2}\left(\left(\omega_{0}, a\right) ; E\right) E:=\mathbf{C}^{2}\right)$ of vector-valued functions using the inner product

$$
(f, g):=\int_{\omega_{0}}^{a}(f(x), g(x))_{E} d_{\omega, q} x .
$$

Next denote by $\mathcal{D}$ the linear set of all vector-valued functions $y \in$ $L_{\omega, q}^{2}\left(\left(\omega_{0}, a\right), E\right)$ such that $y$ are continuous functions at $\omega_{0}$ defined on $\left[\omega_{0}, h^{-1}(a)\right]$, $\Gamma y \in L_{\omega, q}^{2}\left(\left(\omega_{0}, a\right), E\right)$ and satisfying the boundary conditions (3.2), (3.3).

Theorem 1. The boundary-value problem defined by (3.1)-(3.3) is formally self-adjoint on $L_{\omega, q}^{2}\left(\left(\omega_{0}, a\right) ; E\right)$.

Proof. Firstly, we will prove Green's formula. Let $y(),. z(.) \in \mathcal{D}$. Then, we have

$$
\begin{gathered}
(\Gamma y, z)-(y, \Gamma z) \\
=\int_{\omega_{0}}^{a}\left(-q^{-1} D_{--1, q^{-1}} y_{2}+p(x) y_{1}\right) \overline{z_{1}} d_{\omega, q} x \\
+\int_{\omega_{0}}^{a}\left(D_{\omega, q} y_{1}+r(x) y_{2}\right) \overline{z_{2}} d_{\omega, q} x \\
-\int_{\omega_{0}}^{a} y_{1} \overline{\left(-q^{-1} D_{-\omega q^{-1}, q^{-1}} z_{2}+p(x) z_{1}\right)} d_{\omega, q} x \\
-\int_{\omega_{0}}^{a} y_{2} \overline{\left(D_{\omega, q} z_{1}+r(x) z_{2}\right)} d_{\omega, q} x \\
=-\int_{\omega_{0}}^{a}\left[\left(-q^{-1} D_{\left.\left.-\omega q^{-1}, q^{-1} y_{2}\right) \overline{z_{1}}+y_{2} \overline{\left(D_{\omega, q} z_{1}\right)}\right]}\right] d_{\omega, q} x\right. \\
+\int_{\omega_{0}}^{a}\left[\left(D_{\omega, q} y_{1}\right) \overline{z_{2}}+y_{1} \overline{\left(-q^{-1} D_{-\omega q^{-1}, q^{-1}} z_{2}\right)}\right] d d_{\omega, q} x
\end{gathered}
$$

Since

$$
\begin{aligned}
D_{\omega, q}\left(\overline{z_{1}(x)} y_{2}\left(h^{-1}(x)\right)\right) & =\left(D_{\omega, q} y_{2}\left(h^{-1}(x)\right)\right) \overline{z_{1}(x)}+y_{2}(x) \overline{\left(D_{\omega, q} z_{1}(x)\right)} \\
& =\left(-q^{-1} D_{-\omega q^{-1}, q^{-1}} y_{2}\right) \overline{z_{1}}+y_{2} \overline{\left(D_{\omega, q} z_{1}\right)}
\end{aligned}
$$

and 


$$
\begin{aligned}
D_{\omega, q}\left(\overline{z_{2}\left(h^{-1}(x)\right)} y_{1}(x)\right) & =\overline{\left(D_{\omega, q} z_{2}\left(h^{-1}(x)\right)\right)} y_{1}(x)+\overline{z_{2}(x)}\left(D_{\omega, q} y_{1}(x)\right) \\
& =\overline{\left(-q^{-1} D_{-\omega q^{-1}, q^{-1}} z_{2}\right)} y_{1}+\overline{z_{2}(x)} D_{\omega, q} y_{1}(x) .
\end{aligned}
$$

Hence we get

$$
\begin{aligned}
(\Gamma y, z)-(y, \Gamma z)= & -\int_{\omega_{0}}^{a} D_{\omega, q}\left(\overline{z_{1}(x)} y_{2}\left(h^{-1}(x)\right)\right) d_{\omega, q} x \\
& +\int_{\omega_{0}}^{a} D_{\omega, q}\left(y_{1}(x) \overline{z_{2}\left(h^{-1}(x)\right)}\right) d_{\omega, q} x \\
= & \int_{\omega_{0}}^{a} D_{\omega, q}\left[y_{1}(x) \overline{z_{2}\left(h^{-1}(x)\right)}-\overline{z_{1}(x)} y_{2}\left(h^{-1}(x)\right)\right] d_{\omega, q} x .
\end{aligned}
$$

Let us define $[y, z]_{x}:=y_{1}(x) \overline{z_{2}\left(h^{-1}(x)\right)}-\overline{z_{1}(x)} y_{2}\left(h^{-1}(x)\right)$. Hence we obtain

$$
(\Gamma y, z)-(y, \Gamma z)=[y, z]_{a}-[y, z]_{\omega_{0}} .
$$

We proceed to show that the operator $\Gamma$ is formally self-adjoint. It is sufficient to prove that $(\Gamma y, z)=(y, \Gamma z)$ for any $y(),. z(.) \in \mathcal{D}$.

Let $y(),. z(.) \in \mathcal{D}$. Then, we have (3.4), and from the boundary conditions (3.2) and (3.3), we get $[y, z]_{a}=0$ and $[y, z]_{\omega_{0}}=0$.

Consequently,

$$
(\Gamma y, z)=(y, \Gamma z)
$$

This completes the proof.

Lemma 2. All eigenvalues of the problem defined by (3.1)-(3.3) are real.

Proof. Let $\lambda$ be an eigenvalue with an eigenfunction $z$. From (3.5), we get

$$
(\Gamma z, z)=(z, \Gamma z)=(z, \lambda z)=\bar{\lambda}(z, z) .
$$

On the other hand,

$$
(\Gamma z, z)=(\lambda z, z)=\lambda(z, z) .
$$

From (3.6) and (3.7), we obtain $\lambda(z, z)=\bar{\lambda}(z, z)$ or $(\lambda-\bar{\lambda})(z, z)=0$. Since $z \neq 0$, we have $\lambda=\bar{\lambda}$. 
Lemma 3. If $\lambda_{1}$ and $\lambda_{2}$ are two different eigenvalues of the problem defined by (3.1)-(3.3), then the corresponding eigenfunctions $v_{1}$ and $v_{2}$ are orthogonal.

Proof. Let $\lambda_{1}$ and $\lambda_{2}$ be two different real eigenvalues with corresponding eigenfunctions $v_{1}$ and $v_{2}$, respectively. From (3.5), we obtain

$$
\left(\lambda_{1}-\lambda_{2}\right) \int_{\omega_{0}}^{a}\left(v_{1}(x), v_{2}(x)\right)_{E} d_{\omega, q} x=0 .
$$

Since $\lambda_{1} \neq \lambda_{2}$, we obtain that $v_{1}$ and $v_{2}$ are orthogonal.

Theorem 4. Let

$$
y(x)=\left(\begin{array}{l}
y_{1}(x) \\
y_{2}(x)
\end{array}\right), z(x)=\left(\begin{array}{c}
z_{1}(x) \\
z_{2}(x)
\end{array}\right) .
$$

Then, we define the Wronskian of $y(x)$ and $z(x)$ by the formula

$W(y, z)(x)=y_{1}(x) z_{2}\left(h^{-1}(x)\right)-z_{1}(x) y_{2}\left(h^{-1}(x)\right)\left(x \in\left(\omega_{0}, a\right]\right)$. The Wronskian of any solution of Eq. (3.1) is independent of $x\left(x \in\left[\omega_{0}, a\right]\right)$.

Proof. Let $y(x)$ and $z(x)$ be two solutions of Eq. (3.1). By Green's formula (3.4), we have

$$
\int_{\omega_{0}}^{x}\left[(\Gamma y, \bar{z})_{E}-(y, \Gamma \bar{z})_{E}\right](t) d_{\omega, q} t=[y, \bar{z}]_{x}-[y, \bar{z}]_{\omega_{0}} .
$$

Since $\Gamma y=\lambda y$ and $\Gamma \bar{z}=\bar{\lambda} \bar{z}$, we have

$$
\begin{aligned}
\int_{\omega_{0}}^{x}\left[(\lambda y, \bar{z})_{E}-(y, \bar{\lambda} \bar{z})_{E}\right](t) d_{\omega, q} t & =[y, \bar{z}]_{x}-[y, \bar{z}]_{\omega_{0}}, \\
(\lambda-\lambda) \int_{\omega_{0}}^{x}(y(t), \bar{z}(t))_{E} d_{\omega, q} t & =[y, \bar{z}]_{x}-[y, \bar{z}]_{\omega_{0}}=0,
\end{aligned}
$$

or $W(y, z)(x)=[y, \bar{z}]_{x}=[y, \bar{z}]_{\omega_{0}}=W(y, z)\left(\omega_{0}\right)\left(x \in\left(\omega_{0}, a\right]\right)$, i.e., the Wronskian is independent of $x$.

Corollary 5. If $y(x)$ and $z(x)$ are both solutions of Eq. (3.1), then either $W(y, z)=0$ or $W(y, z) \neq 0$ for all $x \in\left[\omega_{0}, a\right]$.

Theorem 6. Any two solutions of Eq. (3.1) are linearly dependent if and only if their Wronskian is zero. 
Proof. Let $y(x)$ and $z(x)$ be two linearly dependent solutions of Eq. (3.1). Then, there exists a constant $c>0$ such that $y(x)=c z(x)$. Hence $\mathrm{W}(y, z)=\left|\begin{array}{ll}y_{1}(x) & y_{2}\left(h^{-1}(x)\right) \\ z_{1}(x) & z_{2}\left(h^{-1}(x)\right)\end{array}\right|=\left|\begin{array}{cc}c z_{1}(x) & c z_{2}\left(h^{-1}(x)\right) \\ z_{1}(x) & z_{2}\left(h^{-1}(x)\right)\end{array}\right|=0$.

Conversely, the Wronskian $W(y, z)=0$ and therefore, $y(x)=c z(x)$, i.e., $y(x)$ and $z(x)$ are linearly dependent.

Lemma 7. All eigenvalues of the problem defined by (3.1)-(3.3) are simple from the geometric point of view.

Proof. Let $\mu$ be an eigenvalue with eigenfunctions $z_{1}(x)$ and $z_{2}(x)$. From the boundary condition (3.2), we have

$\mathrm{W}\left(z_{1}, z_{2}\right)\left(\omega_{0}\right)=z_{11}\left(\omega_{0}\right) z_{22}\left(\omega_{0}\right)-z_{12}\left(\omega_{0}\right) z_{21}\left(\omega_{0}\right)=0$.

Then, the set $\left\{z_{1}(x), z_{2}(x)\right\}$ is linearly dependent.

Now, our next goal is to determine the eigenvalues and the corresponding eigenfunctions. Let

$$
\psi_{1}(x, \lambda)=\left(\begin{array}{l}
\psi_{11}(x, \lambda) \\
\psi_{12}(x, \lambda)
\end{array}\right), \psi_{2}(x, \lambda)=\left(\begin{array}{l}
\psi_{21}(x, \lambda) \\
\psi_{22}(x, \lambda)
\end{array}\right)
$$

be linearly independent solutions of (3.1) which satisfy the initial conditions

$$
\begin{aligned}
& \psi_{11}\left(\omega_{0}, \lambda\right)=1, \psi_{12}\left(\omega_{0}, \lambda\right)=0, \\
& \psi_{21}\left(\omega_{0}, \lambda\right)=0, \psi_{22}\left(\omega_{0}, \lambda\right)=1, \lambda \in \mathbf{C} .
\end{aligned}
$$

Then, every solution of the system (3.1) has the form

$$
y(x, \lambda)=K_{1} \psi_{1}(x, \lambda)+K_{2} \psi_{2}(x, \lambda),
$$

where $K_{1}$ and $K_{2}$ do not depend on $x$. If we can find a nontrivial solution of the linear system

$$
\begin{aligned}
& K_{1} \Gamma_{1}\left(\psi_{1}\right)+K_{2} \Gamma_{1}\left(\psi_{2}\right)=0, \\
& K_{1} \Gamma_{2}\left(\psi_{1}\right)+K_{2} \Gamma_{2}\left(\psi_{2}\right)=0,
\end{aligned}
$$

then the solution $y(x, \lambda)$ is called an eigenfunction of (3.1). Hence $\lambda \in \mathbf{R}$ is an eigenvalue if and only if

$$
\Delta(\lambda)=\left|\begin{array}{ll}
\Gamma_{1}\left(\psi_{1}\right) & \Gamma_{1}\left(\psi_{2}\right) \\
\Gamma_{2}\left(\psi_{1}\right) & \Gamma_{2}\left(\psi_{2}\right)
\end{array}\right|=0
$$

The function $\Delta(\lambda)$ is called the characteristic determinant associated with the Hahn-Dirac system defined by (3.1)-(3.3). The eigenvalues of the problem defined by (3.1)-(3.3) are the zeros of the function $\Delta(\lambda)$. On the 
other hand, $\Delta(\lambda)$ is an entire function in $\lambda$ because $\psi_{1}(x, \lambda)$ and $\psi_{2}(x, \lambda)$ are entire in $\lambda$ for each fixed $x \in\left[\omega_{0}, a\right]$. Hence the eigenvalues of the Hahn-Dirac system defined by (3.1)-(3.3) are at most countable with no finite limit points.

Theorem 8. All eigenvalues $\lambda_{n}(n=1,2, \ldots)$ of the problem defined by (3.1)-(3.3) are simple zeros of the function $\Delta(\lambda)$.

Proof. Let us define

by

$$
\varphi_{1}(x, \lambda)=\left(\begin{array}{l}
\varphi_{11}(x, \lambda) \\
\varphi_{12}(x, \lambda)
\end{array}\right) \text { and } \varphi_{2}(x, \lambda)=\left(\begin{array}{l}
\varphi_{21}(x, \lambda) \\
\varphi_{22}(x, \lambda)
\end{array}\right)
$$

$$
\begin{aligned}
& \varphi_{1}(x, \lambda)=\Gamma_{1}\left(\psi_{2}\right) \psi_{1}(x, \lambda)-\Gamma_{1}\left(\psi_{1}\right) \psi_{2}(x, \lambda), \\
& \varphi_{2}(x, \lambda)=\Gamma_{2}\left(\psi_{2}\right) \psi_{1}(x, \lambda)-\Gamma_{2}\left(\psi_{1}\right) \psi_{2}(x, \lambda) .
\end{aligned}
$$

Then, $\varphi_{1}(x, \lambda)$ and $\varphi_{2}(x, \lambda)$ are solutions of $(3.1)$ such that

$$
\varphi_{1}\left(\omega_{0}, \lambda\right)=\left(\begin{array}{c}
a_{12} \\
-a_{11}
\end{array}\right) \text { and } \varphi_{2}(a, \lambda)=\left(\begin{array}{c}
a_{22} \\
-a_{21}
\end{array}\right) .
$$

On the other hand, we have

$$
\begin{aligned}
& W\left(\varphi_{1}(x, \lambda), \varphi_{2}(x, \lambda)\right) \\
= & \left|\begin{array}{cc}
\varphi_{11}(x, \lambda) & \varphi_{12}\left(h^{-1}(x), \lambda\right) \\
\varphi_{21}(x, \lambda) & \varphi_{22}\left(h^{-1}(x), \lambda\right)
\end{array}\right| \\
= & \varphi_{11}(x, \lambda) \varphi_{22}\left(h^{-1}(x), \lambda\right)-\varphi_{12}\left(h^{-1}(x), \lambda\right) \varphi_{21}(x, \lambda) \\
= & \left(\Gamma_{1}\left(\psi_{2}\right) \psi_{11}(x, \lambda)-\Gamma_{1}\left(\psi_{1}\right) \psi_{21}(x, \lambda)\right) \\
& \times\left(\Gamma_{2}\left(\psi_{2}\right) \psi_{12}(x, \lambda)-\Gamma_{2}\left(\psi_{1}\right) \psi_{22}(x, \lambda)\right) \\
& -\left(\Gamma_{1}\left(\psi_{2}\right) \psi_{12}(x, \lambda)-\Gamma_{1}\left(\psi_{1}\right) \psi_{22}(x, \lambda)\right) \\
& \times\left(\Gamma_{2}\left(\psi_{2}\right) \psi_{11}(x, \lambda)-\Gamma_{2}\left(\psi_{1}\right) \psi_{21}(x, \lambda)\right) \\
= & \Gamma_{1}\left(\psi_{2}\right) \Gamma_{2}\left(\psi_{1}\right)\left(\begin{array}{c}
-\psi_{11}(x, \lambda) \psi_{22}\left(h^{-1}(x), \lambda\right) \\
\left.+h^{-1}(x), \lambda\right) \psi_{21}(x, \lambda)
\end{array}\right) \\
& +\Gamma_{1}\left(\psi_{1}\right) \Gamma_{2}\left(\psi_{2}\right)\left(\begin{array}{c}
\psi_{11}(x, \lambda) \psi_{22}\left(h^{-1}(x), \lambda\right) \\
\left.-h_{12}(x), \lambda\right) \psi_{21}(x, \lambda)
\end{array}\right) \\
= & \left(\psi_{11}(x, \lambda) \psi_{22}\left(h^{-1}(x), \lambda\right)-\psi_{12}\left(h^{-1}(x), \lambda\right) \psi_{21}(x, \lambda)\right) \\
& \times\left(\Gamma_{1}\left(\psi_{1}\right) \Gamma_{2}\left(\psi_{2}\right)-\Gamma_{1}\left(\psi_{2}\right) \Gamma_{2}\left(\psi_{1}\right)\right) \\
(3.10)= & W\left(\psi_{1}(x, \lambda), \psi_{2}(x, \lambda)\right) \Delta(\lambda)=\Delta(\lambda) .
\end{aligned}
$$


Let $\lambda_{0}$ be an eigenvalue of the problem defined by (3.1)-(3.3). Since $\lambda_{0}$ is a real number, $\psi_{i}\left(x, \lambda_{0}\right)(i=1,2)$ can be taken to be real-valued. Then, by $(3.21), \varphi_{1}\left(x, \lambda_{0}\right)$ and $\varphi_{2}\left(x, \lambda_{0}\right)$ are linearly dependent eigenfunctions. Hence there exists a nonzero constant $\eta_{0}$ such that

$$
\varphi_{2}\left(x, \lambda_{0}\right)=\eta_{0} \varphi_{1}\left(x, \lambda_{0}\right) .
$$

From (3.8) and (3.9), we have

$$
\begin{aligned}
& \varphi_{21}\left(\omega_{0}, \lambda_{0}\right)=\eta_{0} a_{12}=\eta_{0} \varphi_{11}\left(\omega_{0}, \lambda\right) \\
& \varphi_{22}\left(\omega_{0}, \lambda_{0}\right)=-\eta_{0} a_{11}=-\eta_{0} \varphi_{12}\left(\omega_{0}, \lambda\right) .
\end{aligned}
$$

If we take $y(x)=\varphi_{2}(x, \lambda)$ and $z(x)=\varphi_{2}\left(x, \lambda_{0}\right)$ in (3.4), then we get

$$
\begin{aligned}
& \left(\lambda-\lambda_{0}\right) \int_{\omega_{0}}^{a}\left(\varphi_{2}(x, \lambda), \varphi_{2}\left(x, \lambda_{0}\right)\right)_{E} d_{\omega, q} x \\
= & -\left(\varphi_{21}\left(\omega_{0}, \lambda\right) \varphi_{22}\left(\omega_{0}, \lambda_{0}\right)-\varphi_{21}\left(\omega_{0}, \lambda_{0}\right) \varphi_{22}\left(\omega_{0}, \lambda\right)\right) \\
= & -\left(\varphi_{21}\left(\omega_{0}, \lambda\right)\left(-\eta_{0} \varphi_{12}\left(\omega_{0}, \lambda\right)\right)-\eta_{0} \varphi_{11}\left(\omega_{0}, \lambda\right) \varphi_{22}\left(\omega_{0}, \lambda\right)\right) \\
= & \eta_{0}\left(\varphi_{11}\left(\omega_{0}, \lambda\right) \varphi_{22}\left(\omega_{0}, \lambda\right)-\varphi_{12}\left(\omega_{0}, \lambda\right) \varphi_{21}\left(\omega_{0}, \lambda\right)\right) \\
= & \eta_{0} W\left(\varphi_{1}(x, \lambda), \varphi_{2}(x, \lambda)\right)=\eta_{0} \Delta(\lambda) .
\end{aligned}
$$

Since $\Delta(\lambda)$ is an entire function in $\lambda$, we have

$$
\frac{d}{d \lambda} \Delta(\lambda):=\lim _{\lambda \rightarrow \lambda_{0}} \frac{\Delta(\lambda)}{\lambda-\lambda_{0}}=\frac{1}{\eta_{0}} \int_{\omega_{0}}^{a}\left\|\varphi_{2}\left(x, \lambda_{0}\right)\right\|_{E}^{2} d_{\omega, q} x \neq 0 .
$$

Consequently, $\lambda_{0}$ is a simple zero of $\Delta(\lambda)$.

\section{Green's function and eigenfunction expansion formula}

In this section, we will study the solution of the nonhomogeneous system

$$
\begin{gathered}
-q^{-1} D_{-\omega q^{-1}, q^{-1}} y_{2}+\{p(x)-\lambda\} y_{1}=f_{1}(x), \\
D_{\omega, q} y_{1}+\{r(x)-\lambda\} y_{2}=f_{2}(x),
\end{gathered}
$$

where $\omega_{0}<x<a<\infty$, which fulfills the boundary conditions

$$
\begin{aligned}
a_{11} y_{1}\left(\omega_{0}\right)+a_{12} y_{2}\left(\omega_{0}\right) & =0, \\
a_{21} y_{1}(a)+a_{22} y_{2}\left(h^{-1}(a)\right) & =0,
\end{aligned}
$$


where $a_{i j} \in \mathbf{R}(i, j=1,2)$ and

$$
\mathrm{f}(.)=\left(\begin{array}{c}
f_{1}(.) \\
f_{2}(.)
\end{array}\right) \in L_{\omega, q}^{2}\left(\left(\omega_{0}, a\right) ; E\right) .
$$

For this, we construct Green's function of the nonhomogeneous system (4.1)-(4.4). We also obtain an eigenfunction expansion for the above system.

Theorem 1. If $\lambda$ is not an eigenvalue of the problem defined by (3.1)-(3.3), then the nonhomogeneous system (4.1)-(4.4) is solvable for any vectorvalued function $f($.$) in L_{\omega, q}^{2}\left(\left(\omega_{0}, a\right) ; E\right)$. Conversely, if $\lambda$ is an eigenvalue of the problem defined by (3.1)-(3.3), then the nonhomogeneous system (4.1)-(4.4) is, generally unsolvable.

Proof. Let us define

$$
G(x, t, \lambda)=\left\{\begin{array}{cc}
-\frac{\varphi_{2}(x, \lambda) \varphi_{1}^{T}(t, \lambda)}{\Delta(\lambda)}, & \omega_{0} \leq t \leq x, \\
-\frac{\varphi_{1}(x, \lambda) \varphi_{2}^{T}(t, \lambda)}{\Delta(\lambda)}, & x<y \leq a
\end{array}\right.
$$

which is called Green's matrix. We will show that the function

$$
y(x, \lambda)=\int_{\omega_{0}}^{a} G(x, t, \lambda) f(t) d_{\omega, q} t
$$

is the solution of the nonhomogeneous system (4.1)-(4.4).

By definition of Green's matrix, we have

$$
\begin{aligned}
& G(x, t, \lambda) \\
& =\left\{\begin{array}{l}
-\frac{1}{\Delta(\lambda)}\left(\begin{array}{ll}
\varphi_{21}(x, \lambda) \varphi_{11}(t, \lambda) & \varphi_{21}(x, \lambda) \varphi_{12}(t, \lambda) \\
\varphi_{22}(x, \lambda) \varphi_{11}(t, \lambda) & \varphi_{22}(x, \lambda) \varphi_{12}(t, \lambda) \\
\varphi_{11}(x, \lambda) \varphi_{21}(t, \lambda) & \varphi_{11}(x, \lambda) \varphi_{22}(t, \lambda) \\
\varphi_{12}(x, \lambda) \varphi_{21}(t, \lambda) & \varphi_{12}(x, \lambda) \varphi_{22}(t, \lambda)
\end{array}\right), \quad \omega_{0} \leq t \leq x, \\
\Delta(\lambda), t \leq a .
\end{array}\right.
\end{aligned}
$$

From (4.6), we have

$$
\begin{gathered}
y_{1}(x, \lambda) \\
=-\frac{1}{\Delta(\lambda)} \varphi_{21}(x, \lambda) \int_{\omega_{0}}^{x}\left(\begin{array}{c}
\varphi_{11}(t, \lambda) f_{1}(t) \\
+\varphi_{12}(t, \lambda) f_{2}(t)
\end{array}\right) d_{\omega, q} t \\
-\frac{1}{\Delta(\lambda)} \varphi_{11}(x, \lambda) \int_{x}^{a}\left(\begin{array}{c}
\varphi_{21}(t, \lambda) f_{1}(t) \\
+\varphi_{22}(t, \lambda) f_{2}(t)
\end{array}\right) d_{\omega, q} t
\end{gathered}
$$




$$
\begin{gathered}
y_{2}(x, \lambda) \\
=-\frac{1}{\Delta(\lambda)} \varphi_{22}(x, \lambda) \int_{\omega_{0}}^{x}\left(\begin{array}{c}
\varphi_{11}(t, \lambda) f_{1}(t) \\
+\varphi_{12}(t, \lambda) f_{2}(t)
\end{array}\right) d_{\omega, q} t \\
-\frac{1}{\Delta(\lambda)} \varphi_{12}(x, \lambda) \int_{x}^{a}\left(\begin{array}{c}
\varphi_{21}(t, \lambda) f_{1}(t) \\
+\varphi_{22}(t, \lambda) f_{2}(t)
\end{array}\right) d_{\omega, q} t .
\end{gathered}
$$

From (4.8), it follows that

$$
\begin{gathered}
D_{\omega, q} y_{1}(x, \lambda) \\
=-\frac{1}{\Delta(\lambda)} D_{\omega, q} \varphi_{21}(x, \lambda) \int_{\omega_{0}}^{x}\left(\begin{array}{c}
\varphi_{11}(t, \lambda) f_{1}(t) \\
+\varphi_{12}(t, \lambda) f_{2}(t)
\end{array}\right) d_{\omega, q} t \\
-\frac{1}{\Delta(\lambda)} D_{\omega, q} \varphi_{11}(x, \lambda) \int_{x}^{a}\left(\begin{array}{c}
\varphi_{21}(t, \lambda) f_{1}(t) \\
+\varphi_{22}(t, \lambda) f_{2}(t)
\end{array}\right) d_{\omega, q} t \\
+\frac{1}{\Delta(\lambda)} W\left(\varphi_{1}, \varphi_{2}\right) f_{2}(x) \\
=\frac{1}{\Delta(\lambda)}\{r(x)-\lambda\} \varphi_{22}(x, \lambda) \int_{\omega_{0}}^{x}\left(\begin{array}{c}
\varphi_{11}(t, \lambda) f_{1}(t) \\
+\varphi_{12}(t, \lambda) f_{2}(t)
\end{array}\right) d_{\omega, q} t \\
\quad \frac{1}{\Delta(\lambda)}\{r(x)-\lambda\} \varphi_{12}(x, \lambda) \int_{x}^{a}\left(\begin{array}{c}
\varphi_{21}(t, \lambda) f_{1}(t) \\
+\varphi_{22}(t, \lambda) f_{2}(t)
\end{array}\right) d_{\omega, q} t \\
+f_{2}(x)=-\{r(x)-\lambda\} y_{2}(x)+f_{2}(x) .
\end{gathered}
$$

The validity of (4.1) is proved similarly. Hence the function $y(x, \lambda)$ in (4.6) is the solution of the system (4.1)-(4.2). We check at once that (4.6) satisfies the boundary conditions (4.3)-(4.4).

Theorem 2. The Green's matrix defined by the formula (4.5) has the following properties:

(i) The Green's matrix $G(x, t, \lambda)$ is unique, i.e., if there exists another Green's matrix $\widetilde{G}(x, t, \lambda)$ for the nonhomogeneous system (4.1)-(4.4), then $G(x, t, \lambda)=\widetilde{G}(x, t, \lambda)$ in $L_{\omega, q}^{2}\left(\left(\omega_{0}, a\right) \times\left(\omega_{0}, a\right) ; E\right)$. 
(ii) $G(x, t, \lambda)$ is continuous at the point $\left(\omega_{0}, \omega_{0}\right)$.

(iii) $G(x, t, \lambda)=G^{T}(t, x, \lambda)$.

(iv) Let $\lambda_{0}$ be a zero of $\Delta(\lambda)$. Then $\lambda_{0}$ can be a simple pole of the matrix $G(x, t, \lambda)$. Therefore, we have

$$
G(x, t, \lambda)=\frac{-v(x) v^{T}(t)}{\lambda-\lambda_{0}}+\widetilde{G}(x, t, \lambda),
$$

where $\widetilde{G}(x, t, \lambda)$ is an analytic function of $\lambda$ in the neighborhood of $\lambda_{0}$ and $v(x)$ is a normalized eigenfunction corresponding to $\lambda_{0}$.

Proof. Since the proof is similar to that of Hahn-Sturm Liouville equations (see [7]), we omit it.

Now we define the operator $\Upsilon: \mathcal{D} \subset L_{\omega, q}^{2}\left(\left(\omega_{0}, a\right), E\right) \rightarrow L_{\omega, q}^{2}\left(\left(\omega_{0}, a\right), E\right)$ as follows. The domain of definition of $\Upsilon$ is $\mathcal{D}$ and we put $\Upsilon y=\Gamma y$ for $y \in \mathcal{D}$.

We next prove the existence of a countable sequence of eigenvalues of $\Upsilon$ with no finite limit points. Later, we will prove that the corresponding eigenfunctions form an orthonormal basis of $L_{\omega, q}^{2}\left(\left(\omega_{0}, a\right) ; E\right)$. Hence we need the following definition and theorems.

Definition 3. A matrix-valued function $M(x, t)$ of two variables with $\omega_{0} \leq x, t \leq a$ is called the $\omega, q$-Hilbert-Schmidt kernel if

$$
\int_{\omega_{0}}^{a} \int_{\omega_{0}}^{a}\|M(x, t)\|_{E}^{2} d_{\omega, q} x d_{\omega, q} t<+\infty .
$$

Theorem 4 ([20]). If

$$
\sum_{i, k=1}^{\infty}\left|a_{i k}\right|^{2}<+\infty
$$

then the operator $A$ defined by the formula

$$
A\left\{x_{i}\right\}=\left\{y_{i}\right\}, i=1,2, \ldots
$$

where

$$
y_{i}=\sum_{k=1}^{\infty} a_{i k} x_{k}, i=1,2, \ldots
$$

is compact in the sequence space $l^{2}$. 
Theorem 5 (Hilbert-Schmidt). Let $A$ be a compact self-adjoint operator mapping a Hilbert space $H$ into itself. Then there is an orthonormal system $\theta_{1}, \theta_{2}, \ldots$ of eigenvectors of $A$, with corresponding nonzero eigenvalues $\lambda_{1}, \lambda_{2}, \ldots$, such that every element $x \in H$ has a unique representation of the form

$$
x=\sum_{n} c_{n} \theta_{n}+x^{\prime}
$$

where $x^{\prime}$ satisfies the condition $A x^{\prime}=0$. Moreover

$$
A x=\sum_{n} \lambda_{n} c_{n} \theta_{n}
$$

and

$$
\lim _{n \rightarrow \infty} \lambda_{n}=0
$$

in the case where there are infinitely many nonzero eigenvalues ([16]).

It is clear that the operator $\Upsilon$ has the same eigenvalues of the HahnDirac problem defined by (3.1)-(3.3). Without loss of generality, we can assume that $\lambda=0$ is not an eigenvalue. Then, $\operatorname{ker} \Upsilon=\{0\}$. Thus the solution of the problem $(\Upsilon y)(x)=f(x), f(x) \in L_{\omega, q}^{2}\left(\left(\omega_{0}, a\right) ; E\right)$ is given by

$$
y(x)=\int_{\omega_{0}}^{a} G(x, t) f(t) d_{\omega, q} t
$$

where

$$
G(x, t)=G(x, t, 0)= \begin{cases}-\frac{\varphi_{2}(x) \varphi_{1}^{T}(t)}{W\left(\varphi_{1}, \varphi_{2}\right)}, & \omega_{0} \leq t \leq x, \\ -\frac{\varphi_{1}(x) \varphi_{2}^{T}(t)}{W\left(\varphi_{1}, \varphi_{2}\right)}, & x<y \leq a .\end{cases}
$$

Theorem 6. $G(x, t)$ defined by (4.13) is a Hilbert-Schmidt kernel.

Proof. By the upper half of the formula (4.13), we have

$$
\int_{\omega_{0}}^{a} d_{\omega, q} x \int_{\omega_{0}}^{x}\|G(x, t)\|_{E}^{2} d_{\omega, q} t<+\infty
$$

and by the lower half of (4.13), we have

$$
\int_{\omega_{0}}^{a} d_{\omega, q} x \int_{x}^{a}\|G(x, t)\|_{E}^{2} d_{\omega, q} t<+\infty
$$


since the inner integral exists and is a linear combination of the products $\varphi_{i j}(x) \varphi_{k l}(t)(i, j, k, l=1,2)$, and these products belong to $L_{\omega, q}^{2}\left(\left(\omega_{0}, a\right) ; E\right)$ because each of the factors belongs to $L_{\omega, q}^{2}\left(\omega_{0}, a\right)$. Then, we obtain

$$
\int_{\omega_{0}}^{a} \int_{\omega_{0}}^{a}\|G(x, t)\|_{E}^{2} d_{\omega, q} x d_{\omega, q} t<+\infty .
$$

Theorem 7. The operator $K$ defined by the formula

$$
(K f)(x)=\int_{\omega_{0}}^{a} G(x, t) f(t) d_{\omega, q} t
$$

is compact and self-adjoint in $L_{\omega, q}^{2}\left(\left(\omega_{0}, a\right) ; E\right)$.

Proof. Let $\psi_{i}=\psi_{i}(t), i=1,2, \ldots$ be a complete, orthonormal basis of $L_{\omega, q}^{2}\left(\left(\omega_{0}, a\right) ; E\right)$. Since $G(x, t)$ is a Hilbert-Schmidt kernel, we can define

$$
\begin{aligned}
x_{i} & =\left(f, \psi_{i}\right)=\int_{\omega_{0}}^{a}\left(f(t), \psi_{i}(t)\right)_{E} d_{\omega, q} t, \\
y_{i} & =\left(g, \psi_{i}\right)=\int_{\omega_{0}}^{a}\left(g(t), \psi_{i}(t)\right)_{E} d_{\omega, q} t, \\
a_{i k} & =\int_{\omega_{0}}^{a} \int_{\omega_{0}}^{a}\left(G(x, t) \psi_{i}(x), \psi_{i}(t)\right)_{E} d_{\omega, q} x d_{\omega, q} t .
\end{aligned}
$$

Then, $L_{\omega, q}^{2}\left(\left(\omega_{0}, a\right) ; E\right)$ is mapped isometrically $l^{2}$. Consequently, our integral operator transforms into the operator defined by the formula (4.12) in the space $l^{2}$ by this mapping, and the condition (4.14) is translated into the condition (4.11). By Theorem 4, this operator is compact. Therefore, the original operator is compact.

Let $f, g \in L_{\omega, q}^{2}\left(\left(\omega_{0}, a\right) ; E\right)$. As $G(x, t)=G^{T}(t, x)$ and $G(x, t)$ is a realvalued matrix function defined on $\left[\omega_{0}, a\right] \times\left[\omega_{0}, a\right]$, we have

$$
\begin{aligned}
(K f, g) & =\int_{\omega_{0}}^{a}((K f)(x), g(x))_{E} d_{\omega, q} x \\
& =\int_{\omega_{0}}^{a} \int_{\omega_{0}}^{a}(G(x, t) f(t), g(x))_{E} d_{\omega, q} t d_{\omega, q} x \\
& =\int_{\omega_{0}}^{a}\left(f(t), \int_{\omega_{0}}^{a} G(x, t) g(x) d_{\omega, q} x\right)_{E} d_{\omega, q} t=(f, K g) .
\end{aligned}
$$


Thus we have proved that $K$ is the self-adjoint operator in $L_{\omega, q}^{2}\left(\left(\omega_{0}, a\right) ; E\right)$.

Theorem 8. The eigenvalues of the operator $\Upsilon$ form an infinite sequence $\left\{\lambda_{n}\right\}_{n=1}^{\infty}$ of real numbers which can be ordered so that

$$
\left|\lambda_{1}\right|<\left|\lambda_{2}\right|<\ldots<\left|\lambda_{n}\right|<\ldots \rightarrow \infty \text { as } n \rightarrow \infty .
$$

The set of all normalized eigenfunctions of $\Upsilon$ forms an orthonormal basis for $L_{\omega, q}^{2}\left(\left(\omega_{0}, a\right) ; E\right)$.

Proof. By Theorems 5 and 7, the operator $K$ has an infinite sequence of non-zero real eigenvalues $\left\{\xi_{n}\right\}_{n=1}^{\infty}$ with $\lim _{n \rightarrow \infty} \xi_{n}=0$. Then,

$$
\left|\lambda_{n}\right|=\frac{1}{\left|\xi_{n}\right|} \rightarrow \infty \text { as } n \rightarrow \infty .
$$

Furthermore, let $\left\{\chi_{n}\right\}_{n=1}^{\infty}$ denote an orthonormal set of eigenfunctions corresponding to $\left\{\xi_{n}\right\}_{n=1}^{\infty}$. Let $y \in \mathcal{D}$, then $\Upsilon y=f$ and $y=K f$ for some $f \in L_{\omega, q}^{2}\left(\left(\omega_{0}, a\right) ; E\right)$. So

$$
\begin{aligned}
y & =K f=\sum_{n=1}^{\infty} \xi_{n}\left(f, \chi_{n}\right) \chi_{n}=\sum_{n=1}^{\infty} \xi_{n}\left(\Upsilon y, \chi_{n}\right) \chi_{n} \\
& =\sum_{n=1}^{\infty} \xi_{n}\left(y, \Upsilon \chi_{n}\right) \chi_{n}=\sum_{n=1}^{\infty} \xi_{n}\left(y, \lambda_{n} \chi_{n}\right) \chi_{n}=\sum_{n=1}^{\infty}\left(y, \chi_{n}\right) \chi_{n} .
\end{aligned}
$$




\section{References}

[1] B. P. Allahverdiev and H. Tuna, "One-dimensional q-Dirac equation", Mathematical methods in the applied sciences, vol. 40, no. 18, pp. 7287-7306, 2017.

[2] B. P. Allahverdiev and H. Tuna, "The spectral expansion for the Hahn-Dirac system on the whole line", Turkish journal of mathematics, vol. 43, pp. 1668-1687, 2019.

[3] B. P. Allahverdiev and H. Tuna, "The Parseval equality and expansion formula for Singular Hahn-Dirac system," in Emerging applications of differential equations and game theory, S. Z. Alparslan Gök and D. Aruğaslan Çinçin, Eds. Hershey, PA: IGI Global, 2019, pp. 209-235.

[4] K. Aldwoah, "Generalized time scales and associated difference equations", Ph.D. Thesis, Cairo University, 2009.

[5] R. Álvarez-Nodarse, "On characterizations of classical polynomials", Journal of computational and applied mathematics, vol. 196, no. 1, pp. 320-337, 2006.

[6] M. H. Annaby, A. E. Hamza, and K. A. Aldwoah, "Hahn difference operator and associated Jackson-Nörlund integrals", Journal of optimization theory and applications, vol. 154, pp. 133-153, 2012.

[7] M. H. Annaby, A. E. Hamza, and S. D. Makharesh, "A Sturm-Liouville theory for Hahn difference operator", in Frontiers of orthogonal polynomials and q-series, X. Li and Z. Nashed, Eds. Singapore: World Scientific, pp. 35-84, 2018.

[8] A. Dobrogowska and A. Odzijewicz, "Second order q-difference equations solvable by factorization method", Journal of computational and applied mathematics, vol. 193, no. 1, pp. 319-346, 2006.

[9] W. Hahn, "Uber orthogonalpolynome, die q-differenzengleichungen genügen", Mathematische nachrichten, vol. 2, no. 1-2, pp. 4-34, 1949.

[10] W. Hahn, "Ein beitrag zur theorie der orthogonalpolynome", Monatshefte für mathematik, vol. 95, no. 1, pp. 19-24, 1983.

[11] A. E. Hamza and S. A. Ahmed, "Theory of linear Hahn difference equations", Journal of advances in mathematics, vol. 4, no. 2, pp. 440-460, 2013. 
[12] A. E. Hamza and S. A. Ahmed, "Existence and uniqueness of solutions of Hahn difference equations", Advances in difference equations, vol. 2013, Art. ID. 316, 2013.

[13] A. E. Hamza and S. D. Makharesh, "Leibniz's rule and Fubinis theorem associated with Hahn difference operator", Journal of advanced mathematical, vol. 12, no. 6, pp. 6335-6345, 2016.

[14] F. Hira, "Dirac system associated with Hahn difference operator", Bulletin of the Malaysian Mathematical Sciences Society, vol. 43, pp. 3481-3497, 2020.

[15] F. H. Jackson, "q-Difference equations”, American journal of mathematics, vol. 32, no. 4, pp. 305-314, 1910.

[16] A. N. Kolmogorov and S. V. Fomin, Introductory real analysis. New York, NY: Dover, 1970.

[17] K. H. Kwon, D. W. Lee, S. B. Park, and B. H. Yoo, "Hahn class orthogonal polynomials", Kyungpook mathematical journal, vol. 38, pp. 259-281, 1998.

[18] P. Lesky, Eine charakterisierung der Klassischen kontinuierlichen-, diskreten- und Q-orthogonalpolynome. Aachen: Shaker, 2005.

[19] B. M. Levitan and I. S. Sargsjan, Sturm-Liouville and Dirac operators. Dordrecht: Kluwer, 1991.

[20] M. A. Naĭmark, Linear differential operators, 2 vols. New York, NY: F. Ungar, 1969.

[21] J. Petronilho, "Generic formulas for the values at the singular points of some special monic classical Hq,w-orthogonal polynomials", Journal of computational and applied mathematics, vol. 205, no. 1, pp. 314-324, 2007.

[22] T. Sitthiwirattham, "On a nonlocal boundary value problem for nonlinear second-order Hahn difference equation with two different q, $\omega$-derivatives", Advances in difference equations, vol. 116, no. 1, Art. ID. 116, 2016.

[23] B. Thaller, The Dirac equation. Berlin: Springer, 1992.

[24] J. Weidmann, Spectral theory of ordinary differential operators. Berlin: Springer, 1987. 


\section{B. P. Allahverdiev}

Department of Mathematics,

Süleyman Demirel University, 32260 Isparta

Turkey

e-mail: bilenderpasaoglu@sdu.edu.tr

and

\section{Hüseyin Tuna}

Department of Mathematics,

Mehmet Akif Ersoy University,

15030 Burdur

Turkey

e-mail: hustuna@gmail.com

Corresponding author 